\title{
Modification of the loops in the ligand-binding site turns avidin into a steroid-binding protein
}

\author{
Tiina A Riihimäki', Soili Hiltunen ${ }^{1}$, Martina Rangl², Henri R Nordlund', Juha AE Määttä', Andreas Ebner², \\ Peter Hinterdorfer ${ }^{2}$, Markku S Kulomaa ${ }^{1}$, Kristiina Takkinen ${ }^{3}$ and Vesa P Hytönen ${ }^{1 *}$
}

\begin{abstract}
Background: Engineered proteins, with non-immunoglobulin scaffolds, have become an important alternative to antibodies in many biotechnical and therapeutic applications. When compared to antibodies, tailored proteins may provide advantageous properties such as a smaller size or a more stable structure.

Results: Avidin is a widely used protein in biomedicine and biotechnology. To tailor the binding properties of avidin, we have designed a sequence-randomized avidin library with mutagenesis focused at the loop area of the binding site. Selection from the generated library led to the isolation of a steroid-binding avidin mutant (sbAvd-1) showing micromolar affinity towards testosterone $\left(K_{d} \sim 9 \mu \mathrm{M}\right)$. Furthermore, a gene library based on the sbAvd-1 gene was created by randomizing the loop area between $\beta$-strands 3 and 4 . Phage display selection from this library led to the isolation of a steroid-binding protein with significantly decreased biotin binding affinity compared to sbAvd-1. Importantly, differential scanning calorimetry and analytical gel-filtration revealed that the high stability and the tetrameric structure were preserved in these engineered avidins.
\end{abstract}

Conclusions: The high stability and structural properties of avidin make it an attractive molecule for the engineering of novel receptors. This methodology may allow the use of avidin as a universal scaffold in the development of novel receptors for small molecules.

Keywords: protein engineering, avidin scaffold, phage display, steroid hormone, testosterone

\section{Background}

Antibodies are the most widely used biomolecules for therapeutic, diagnostic and research applications, because they can be generated against virtually any molecule using protein engineering techniques (for a review see [1]). However, antibodies have certain fundamental disadvantages such as the complex architecture of their antigen-binding site, low stability, and a rather large size [2-5]. Moreover, the production of full-size antibodies is relatively expensive [6]. To overcome these limitations and to improve therapeutic antibodies, antibodies have been extensively engineered [7]. For example the size of the antibody molecule has been reduced by producing single-domain antigen-binding derivatives [8]. In addition to extensive antibody engineering, a

\footnotetext{
* Correspondence: vesa.hytonen@uta.fi

'Institute of Biomedical Technology, University of Tampere and Tampere University Hospital, FI-33520 Tampere, Finland Full list of author information is available at the end of the article
}

versatile repertoire of tailored biomolecules from nonimmunoglobulin protein scaffolds have been generated $[4,9,10]$. Anticalins, derived from the lipocalin fold, are a good example of engineered proteins [11]. The $\beta$-barrel structure of lipocalins is thermostable and robust and serves as an excellent scaffold for engineering novel receptors. They have been modified to bind novel ligands, such as fluorescein and digoxigenin, with affinities comparable with antibodies [12].

Chicken avidin (Avd), known for its extremely high affinity towards the water-soluble vitamin $\mathrm{H}$, D-biotin, has been widely used in life science research applications [13]. Aside from biotin, Avd also binds dyes and peptides, which share no significant structural similarity with biotin $[14,15]$. Avd provides an attractive robust scaffold for the development of novel receptors, and Avd has many advantageous properties such as high chemical and thermal stability, a deep ligand binding site optimized for the binding of small molecules, and
C Biomed Central 
an oligomeric nature enabling signal amplification. Moreover, the structure of Avd is well characterized $[16,17]$ and numerous engineered forms of Avd have been described [18]. Engineered Avd forms, in which the two pairs of the binding sites (dual-chain Avd) [19-21] or all four binding sites (single-chain Avd) [22] can be independently manipulated, have been developed.

In the present study Avd was modified to bind steroid hormones. Avd mutant sbAvd-1, which was captured using the phage display selection [23], has a micromolar affinity to testosterone. The steroid-binding protein sbAvd-1 was characterized and further engineered to decrease cross-reactivity towards other molecules, especially towards Avd's natural ligand biotin. The resulting sbAvd- 2 mutant with a modified loop between $\beta$-strands 3 and 4 was found to prefer steroid hormones over biotin in ligand binding.

\section{Results}

\section{Functional display of Avd protein on the M13 phage}

Avd was displayed on the surface of the M13 phage as a fusion with the $\mathrm{C}$-terminal region of the minor coat protein pIII. Two different strategies for displaying the Avd scaffold in the active form on the M13 phage were evaluated. In the first display construct, Avd was produced solely as a fusion with pIII (Avd-pIII; Figure 1A), whereas in the Avd/Avd-pIII display construct, free Avd subunits were produced in addition to the pIII fusion (Figure 1B).

The size and oligomeric state of the Avd-pIII fusions were analyzed by SDS-PAGE and by western blot. Polyclonal anti-Avd (Figure 2A) indicated expression of both the Avd-pIII fusion (upper arrow, $38 \mathrm{kDa}$ ) and the free Avd (lower arrow, $\sim 15 \mathrm{kDa}$ ). The production of free subunits should enhance the functional assembly of the tetrameric Avd scaffold, especially if membrane anchoring of Avd by the pIII fusion partner has a negative effect on the oligomerization of Avd subunits. This strategy mimics the generally used amber-stop codon technique, in which free subunits are produced by the

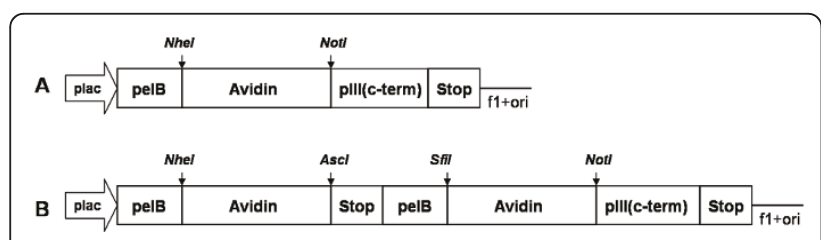

Figure 1 Schematic presentation of the Avd display expression constructs. (A) The phagemid constructs for Avd and Avd(N118M) display, in which Avd protein is produced solely as a fusion with plll. (B) The phagemid constructs for Avd/Avd-plll and Avd(N118M)/ Avd(N118M)-plll display, in which the pelB signal sequence is used for secretion of the Avd-plll fusions and the free Avd. The cloning sites used are shown by vertical arrows.

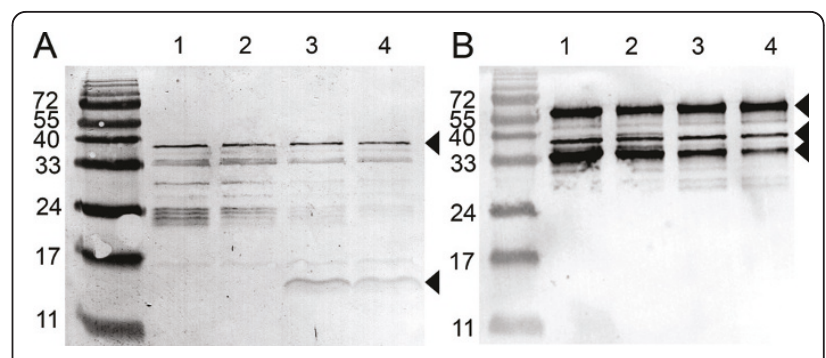

Figure 2 Immunoblot analysis of Avd phages with anti-Avd and anti-plll antibodies. (A) The location of the Avd-plll fusion protein recognized by anti-Avd is indicated by the upper arrowhead. The theoretical mass of the Avd-plll fusion protein is 38 $\mathrm{kDa}$. The produced free Avd $(\sim 14 \mathrm{kDa})$ is indicated with the lower arrowhead. (B) From the immunoblot analyzed with anti-plll antibody the full-length plll expressed from the helper phage (VCSM13) can be seen migrating at $\sim 60 \mathrm{kDa}$ (the upper arrowhead). The Avd-plll fusion is indicated by the middle arrowhead. In addition, some proteolytically truncated Avd-plll forms were detected ( 33 kDa; lower arrowhead). Lane 1: Avd-plll phage; lane 2: Avd(N118M)-plll phage; lane 3: Avd/Avd-plll phage; lane 4: Avd (N118M)/Avd(N118M)-plll phage. The molecular weights of standard proteins are shown as kilodaltons on the left side of each blot.

read-through of amber stop codon by the tRNA. We assume that the oligomerization of Avd, secreted by the pelB signal sequence, occurs in the periplasmic space of $E$. coli, allowing the display of functional Avd on the phage, as is the case for the display of the antibody Fab fragment, which requires folding of the heavy and light chains for the assembly of a functional antibody molecule [24].

A portion of the Avd-pIII fusion was partially proteolytically cleaved, as can be seen from the blot analyzed with the anti-pIII monoclonal antibody (Figure 2B, 35 $\mathrm{kDa}$ band). Because Avd display constructs designed in this study were based on the monovalent display mode $(3+3)$, the intact pIII (migrating at $\sim 58 \mathrm{kDa}$ ) expressed from the helper phage was also detected from the blot (Figure 2B, uppermost arrow).

Avd-displaying phages were functional because they bound specifically to the biotin-coated surfaces, and phages were efficiently amplified even after several panning rounds. Moreover, phagemid DNA with the Avd display expression unit, which was confirmed by the restriction enzyme digestion analysis, was stable during the panning rounds (data not shown).

To analyze the functionality of phages, the mixture of amplified Avd and Avd mutant N118M phages were screened by panning against 4-hydroxyazobenzene-2carboxylic acid (HABA). As determined in our previous study, the biotin binding affinity of the Avd mutant $\mathrm{N} 118 \mathrm{M}$ was reduced $\sim 1,000,000$-fold $\left(\mathrm{K}_{\mathrm{d}}=4.2 \times 10^{-9}\right.$ $\mathrm{M})$ and HABA-affinity was increased $\sim 1.5$-fold $\left(\mathrm{K}_{\mathrm{d}}=5.2\right.$ $\times 10^{-6} \mathrm{M}$ ) compared to wtAvd [25] (Additional file 1). During the panning procedure a clear enrichment of 
Avd(N118M) phages over wt Avd phages was detected, which was an indication of the high selectivity of the produced phages. After only three rounds of selection phages displaying the Avd mutant N118M outcompeted the wtAvd phage population (Additional file 2).

\section{Capture of a steroid-binding avidin}

The loops adjacent to the ligand-binding site were selected for randomization [16]. First we created a library (Avd L1,2 library) in which residues N12, D13, L14, G15, and S16 were randomized. These amino acids form a loop between $\beta$-strands 1 and 2 (Figure 3). Codon NNN was used for randomization, and therefore, all 20 different amino acids were present, including all stop codons. The Avd L1,2 library was ligated into a phagemid as a sole fusion with the $\mathrm{C}$-terminal portion of pIII. Based on sequencing results and transformation efficiency, the Avd L1,2 library was found to consist of approximately $1 \times 10^{5}$ individual members. When a stretch of five amino acid residues is completely randomized, the theoretical library size is $3.2 \times 10^{6}$.

In the panning experiments Avd L1,2 loop library phages were introduced onto a testosterone-coated surface. The phage genomes carrying the mutated cDNA were found to be stable during the selection. A clear enrichment of sequences in the randomized loop area was observed, indicating the success of the selection conditions. Interestingly, we detected N12 as being a highly conserved amino acid residue among the

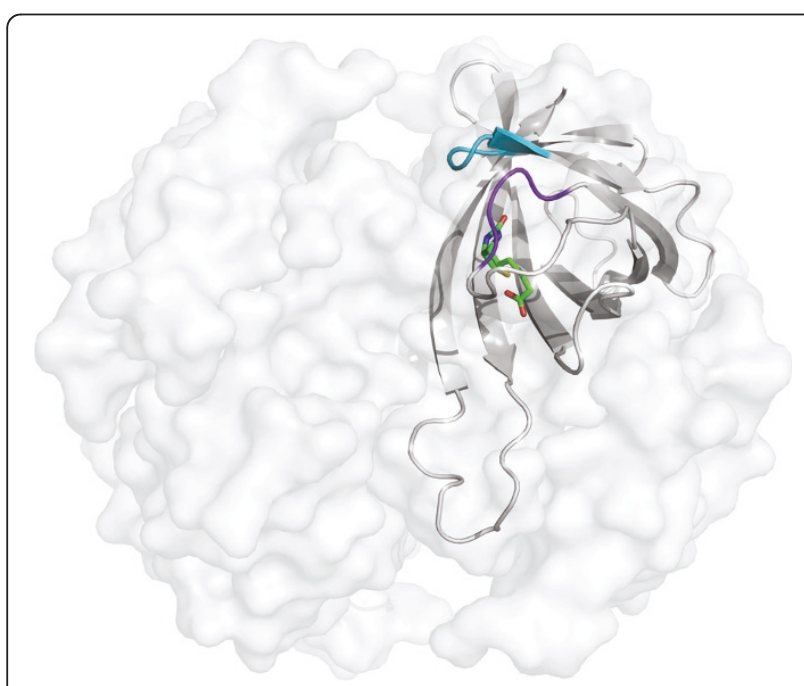

Figure 3 Three-dimensional structure of wtAvd with the loops chosen for random mutagenesis marked. One subunit of tetrameric wtAvd with bound biotin (PDB: 2AVI) [16] is shown in the figure. The randomized amino acid residues are N12, D13, L14, G15, and S16 (shown in blue) in the loop between $\beta$-strands 1 and 2 , and T35, A36, V37, and T38 (shown in purple) in the loop between $\beta$-strands 3 and 4 . enriched pool of proteins. An Avd variant, named sbAvd-1, that carried the sequence N12, R13, M14, N15, H16 was selected for further analysis.

\section{The specificity of sbAvd-1 can be tuned by additional mutations in the loop between $\beta$-strands 3 and 4}

To further lower the biotin-binding affinity and to decrease the cross-reactivity of steroid-binding Avd, a library (sbAvd-1 L3,4) was generated in which the loop area between $\beta$-strands 3 and 4 was randomized (Figure 3 ). The loop between $\beta$-strands 3 and 4 is highly important for biotin binding of Avd because this loop 'locks' biotin into the binding site. In this process, three amino acid residues in the loop form direct interactions with biotin [16]. In the library four amino acids (T35, A36, V37 and, T38) were randomized using the NNY codon. The use of this codon covers 14 of the 20 naturally occurring amino acids while eliminating all of the stop codons. The library consisted of approximately $1.4 \times 10^{6}$ individual members, when calculated from the sequencing results and transformation efficiency, which exceeds the theoretical size of the library calculated based on the possible combinations of amino acid residues $\left(3.8 \times 10^{4}\right)$.

Binders from the sbAvd-1 L3,4 library were selected by phage display panning against a testosterone surface. In every panning round washes were adjusted according to the number of output colonies. The quality of the phage genomes carrying the mutated cDNA was evaluated by DNA sequencing at various stages during selection. A combination of acid and testosterone was used for elution. The selected sbAvd-1 phage clones were evaluated by microplate analysis using BSA-testosterone as a target ligand and utilizing M13-antibody to determine the amount of bound phages. The sbAvd-1 variant that showed the highest binding activity in the microplate assay (data not shown) had the sequence A35, T36, V37, N38. This mutant, named sbAvd-2 was selected for comparative analysis with sbAvd-1.

\section{Production and purification of Avd mutants}

Proteins were produced in soluble form in the $E$. coli strain BL21-AI using N-terminal OmpA bacterial secretion signal from Bordetella avium [26]. Proteins were purified by Ni-NTA affinity chromatography that yielded $\sim 2 \mathrm{mg} / \mathrm{L}$ pure protein. According to gelfiltration analysis, in solution, both sbAvd-1 (51 kDa) and sbAvd-2 (53 kDa) showed tetrameric state (Additional file 3) similar to that of wtAvd (Avd expressed in E. coli $53 \mathrm{kDa}$, chicken Avd $60 \mathrm{kDa}$ [26]). The slight decrease in molecular weight of Avd expressed in bacteria can be explained by the lack of glycosylation. 

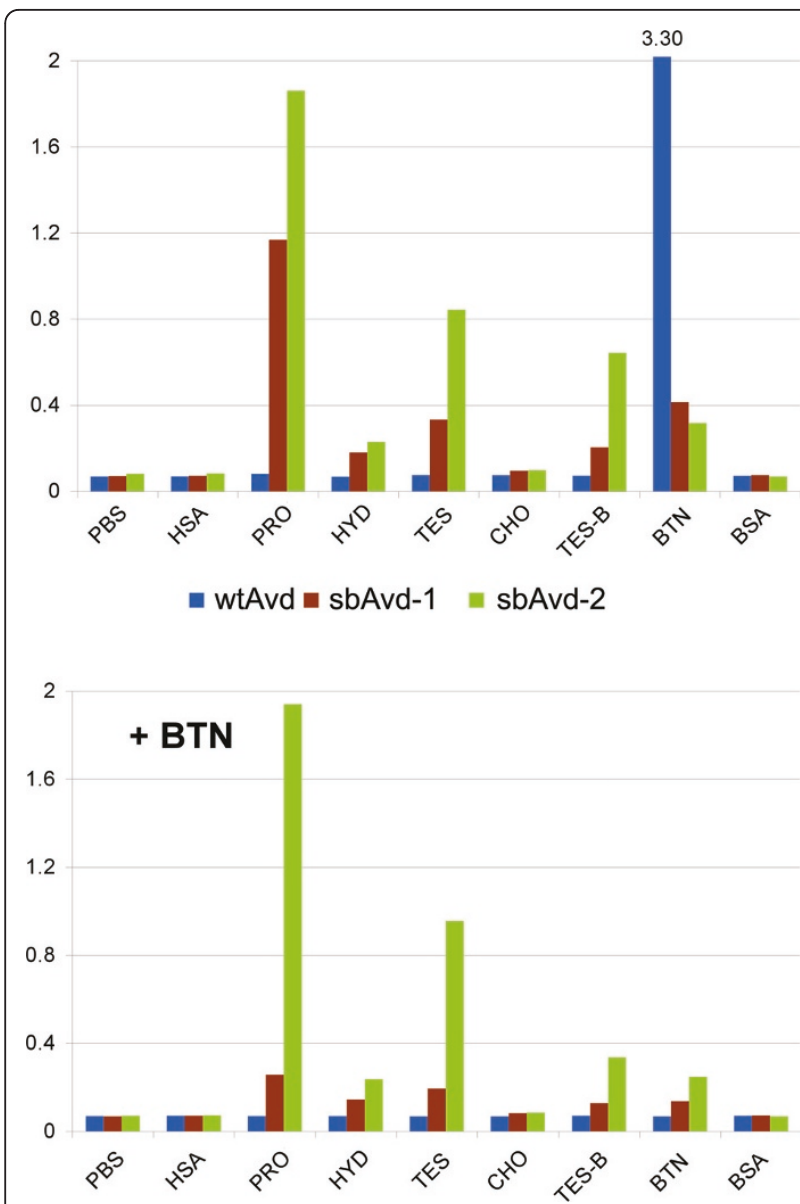

Figure 4 Determination of ligand-binding specificity of sbAvd1 and sbAvd-2 proteins by microplate analysis. The binding of sbAvd-1 and sbAvd-2 to a set of different small ligands was detected using polyclonal anti-avidin antibody as a probe (A) and the effect of free biotin $(10 \mu \mathrm{M})$ to ligand-binding was analyzed (B). Abbreviations used in the figure: PBS, Phosphate buffered saline: HSA, human serum albumin; PRO, HSA-conjugated progesterone; HYD, HSA-conjugated hydrocortisone; TES, HSA-conjugated testosterone; CHO, HSA-conjugated cholic acid; TES-B, BSAconjugated testosterone; BTN, BSA-conjugated D-biotin; BSA, bovine serum albumin

\section{Determination of ligand-binding specificity of Avd forms} by microplate assay

The binding specificity of proteins was analyzed by microplate assay, in which a set of different small molecules were used as a target molecules (Figure 4A). WtAvd was used as a negative control, and we detected no affinity towards the ligands except biotin. Importantly, sbAvd-1 and sbAvd-2 did not bind the proteins used as carriers for small molecules (bovine serum albumin (BSA) and human serum albumin (HSA)). However, these modified Avds showed clear binding to testosterone and progesterone.

The binding to the surface-immobilized ligands was inhibited by pre-incubating the proteins with 10 $\mu \mathrm{M}$ D-biotin (Figure 4B). Free biotin significantly inhibited the binding of sbAvd-1 to steroids, indicating notable affinity towards biotin. However, in the case of sbAvd-2 the binding of testosterone and progesterone was not affected by biotin, showing a clear decrease in biotin-binding affinity.

\section{Biosensor analyses of steroid-binding Avds}

The kinetic constants of testosterone- and biotin-binding to steroid-binding proteins were determined with surface plasmon resonance (SPR) analysis. The purified sbAvd-1 and sbAvd-2 bound to a testosterone-BSAcoated sensor chip with similar affinities (Table 1), whereas wtAvd showed no binding to the testosterone surface. Furthermore, testosterone-binding was inhibited with varying testosterone concentrations $(0.75-50 \mu \mathrm{M}$, data not shown). In the case of sbAvd-1, the $50 \%$ inhibition was achieved between 5-10 $\mu \mathrm{M}$ testosterone, which is consistent with the determined testosterone surface binding affinity. Interestingly, the $50 \%$ inhibition was already achieved in $750 \mathrm{nM}$ testosterone concentration in the sbAvd-2 measurements. This result suggests a much higher binding affinity towards free testosterone than that measured towards surface-immobilized testosterone.

The biotin binding of the steroid-binding proteins was analyzed by the biotin-coated sensor chip. The comparison of sbAvd-1 and sbAvd-2 revealed that the biotinbinding affinity decreased almost 500 -fold due to the modification of the loop between $\beta$-strands 3 and 4 (Table 1). This result appears to be consistent with the previous study in which mutation of $\mathrm{T} 35 \mathrm{~A}$ alone decreased the biotin-binding affinity of wtAvd approximately 200-fold [20].

The specificity of the steroid-binding was analyzed by binding competition using the following steroids: testosterone, dehydroepiandrosterone sulfate (DHEAS), androstenedione, estradiol, and dihydrotestosterone (DHT). In addition, the binding to the surface was also competed for by biotin. SbAvd-1 was found to be cross-reactive with androgens highly similar to testosterone (DHEAS and androstenedione), and had noticeable affinity towards biotin (Figure 5A). Interestingly, DHT showed less efficient inhibition compared to testosterone, suggesting lower binding affinity towards this steroid form. The binding of sbAvd-2 to testosterone was found to be most efficiently inhibited by testosterone and DHEAS (Figure $5 \mathrm{~B})$, whereas the inhibition caused by biotin was clearly weaker than that in the case of sbAvd-1. This result is consistent with the microplate analysis results and with the binding kinetic constants determined with SPR analysis. 
Table 1 Kinetic parameters and the determined thermostability of sbAvds

\begin{tabular}{|c|c|c|c|c|c|c|c|}
\hline \multirow[t]{2}{*}{ Protein } & \multirow[t]{2}{*}{ Ligand } & \multicolumn{2}{|l|}{ SPR } & \multicolumn{3}{|c|}{ DSC } & \multirow[b]{2}{*}{$\Delta \mathrm{H} \times 10^{4}(\mathrm{cal} / \mathrm{mol})$} \\
\hline & & $k_{a}(1 / M s)$ & $k_{d}(1 / s)$ & $\mathrm{K}_{\mathrm{D}}(\mathrm{M})$ & $\mathrm{T}_{\mathrm{m}}\left({ }^{\circ} \mathrm{C}\right)$ & $\Delta \mathrm{T}_{\mathrm{m}}\left({ }^{\circ} \mathrm{C}\right)$ & \\
\hline wtAvd & - & - & - & - & 85.5 & - & 5.4 \\
\hline wtAvd & Btn & n.d. & n.d. & n.d. & 123.2 & 37.7 & 12.8 \\
\hline wtAvd & Tes & - & - & no binding & 86.2 & 0.7 & 5.6 \\
\hline sbAvd-1 & - & - & - & - & 80.6 & - & 5.2 \\
\hline sbAvd-1 & Btn & $4.2 \times 10^{5}$ & $5.6 \times 10^{-4}$ & $1.4 \times 10^{-9}$ & 83.2 & 2.6 & 6.4 \\
\hline sbAvd-1 & Tes & $1.0 \times 10^{3}$ & $9.5 \times 10^{-3}$ & $9.0 \times 10^{-6}$ & 81.5 & 0.9 & 5.7 \\
\hline sbAvd-2 & - & - & - & - & 82.5 & - & 4.6 \\
\hline sbAvd-2 & Btn & $1.0 \times 10^{3}$ & $6.8 \times 10^{-4}$ & $6.6 \times 10^{-7}$ & 83.0 & 0.5 & 4.2 \\
\hline sbAvd-2 & Tes & 813 & $8.5 \times 10^{-3}$ & $1.1 \times 10^{-5}$ & 83.1 & 0.6 & 4.8 \\
\hline
\end{tabular}

The kinetic parameters determined by SPR analysis. The biotin binding affinity of avidin is too high to be determined by SPR. The transition midpoint of thermal unfolding $\left(T_{m}\right)$ and the calorimetric heat of unfolding were determined by DSC. Delta $T_{m}$ represents the increase of $T_{m}$ in the presence of $50 \mu M$ ligand.

\section{Interaction analysis of steroid-binding Avds by MRFS}

Molecular recognition force spectroscopy (MRFS) [27] was used to study the interaction between sbAvds and testosterone on a single molecule level. An atomic force microscopy (AFM) tip was functionalized with a single testosterone molecule [28] and repeatedly approached and retracted from the sbAvd-1- or sbAvd-2-coated surface (Figure 6A). The binding forces were measured in force-distance cycles, whereby the deflection (force) of the cantilever was recorded as a function of the tip-sample distance. For evaluation, 1,000 force-distance cycles were recorded, and probability density functions (pdf) were generated from the detected interaction forces [29]. The tip-tethered testosterone was found to form a complex with the sbAvds; the retraction led to a downward bending of the cantilever until a particular force was reached resulting in the rupture of the bond between testosterone and sbAvd (Figure 6B and 6C). The most probable unbinding force [29] was found to be similar for both of the testosterone-sbAvd complexes: $40 \mathrm{pN}$ at a constant pulling velocity of $600 \mathrm{~nm} / \mathrm{s}$. From 1,000 recorded force-distance cycles, sbAvd-1 showed 183 detected interactions and sbAvd-2 showed 215 events.

To prove the specificity of the measured interactions, control experiments were performed. For these experiments, free testosterone was injected into the measuring solution to preoccupy the binding sites of the steroidbinding protein immobilized on the surface. The retraction curve identical to the approaching curve was

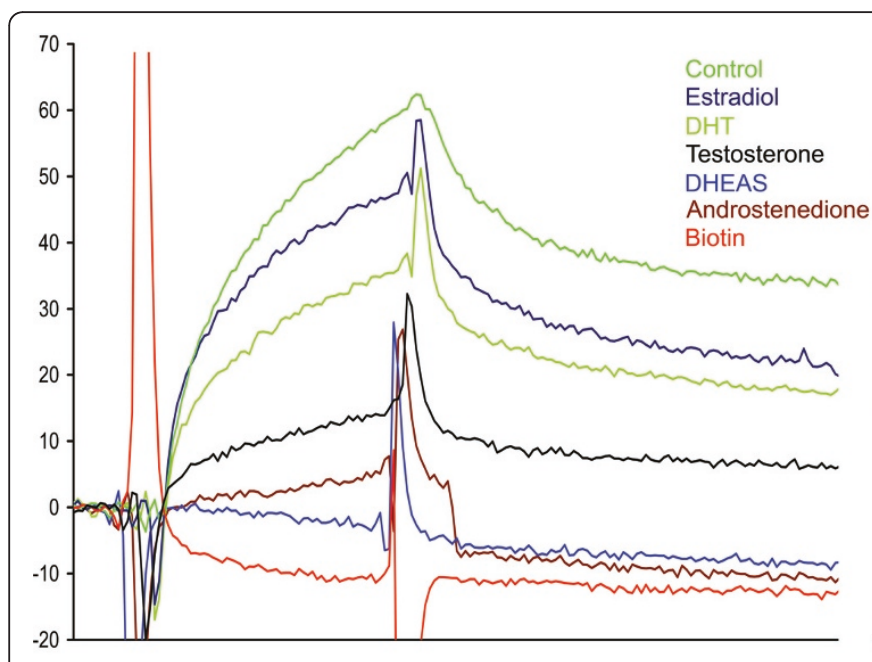

A

Figure 5 Inhibition analysis of sbAvd-1 and sbAvd-2 proteins by the SPR method. The binding of the sbAvd-1 and the sbAvd-2 to a CM5 sensor chip functionalized with testosterone-BSA was measured in the presence of $50 \mu \mathrm{M}$ inhibitors. (A) The binding of the sbAvd-1 protein was totally inhibited by dehydroepiandrosterone, androstenedione, and biotin. (B) The binding of the sbAvd-2 protein was totally inhibited by dehydroepiandrosterone or testosterone, but not biotin. This result is due to the markedly decreased affinity of the protein towards biotin. Samples: Protein sample, green; protein with estradiol, dark blue; protein with DHT, olive; protein with testosterone, black; protein with DHEAS, blue; protein with androstenedione, brown; protein with biotin, red. 


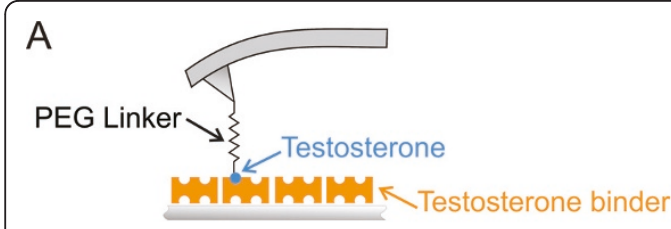

B
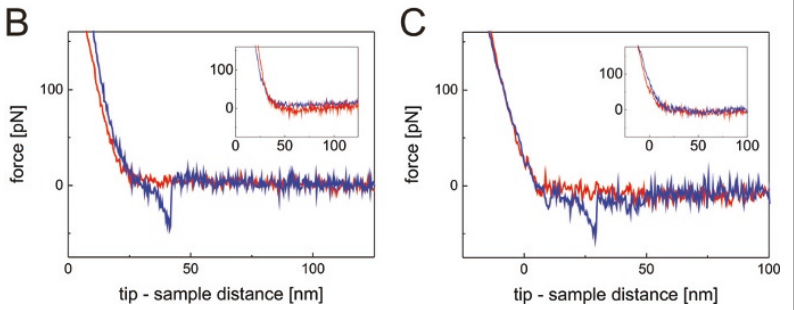

Figure 6 Analysis of sbAvd protein-testosterone interaction by MRFS method (A) A schematic representation of the experimental assembly used in the analyses. Testosterone was tethered to the AFM tip using a flexible PEG crosslinker. The steroid-binders sbAvd-1 and sbAvd-2 were covalently bound to the mica surface via a short homobifunctional spacer. (B) The force-distance cycle of the sbAvd1 - testosterone interaction showing an unbinding event. The typical non-linear shape of the event results from the elastic properties of the PEG linker. (C) The force-distance cycle showing a sbAvd-2 - testosterone bond dissociation. Insets in (B) and (C) represent force-distance cycles in which the protein-testosterone interaction is inhibited with free testosterone.

detected (insets of Figure 6B and 6C), which indicated the total inhibition of the binding by free testosterone. Additionally, when the binding sites were preoccupied with free testosterone, the number of binding events dropped down to 67 from 183 detected interactions in the case of sbAvd-1. In case of sbAvd-2 the number of interactions dropped from of 215 to 21 after addition of free testosterone.

\section{Differential scanning calorimetry reveals the high stability of the engineered proteins}

The high thermal stability of wtAvd (temperature transition midpoint $\left.\left(\mathrm{T}_{\mathrm{m}}\right)=85.5^{\circ} \mathrm{C}\right)$ was preserved in the steroid-binding mutants; these proteins showed similar $T_{m}$ values compared to wtAvd (sbAvd-1 $\mathrm{T}_{\mathrm{m}}$ value of $80.6^{\circ} \mathrm{C}$ and sbAvd-2 $\mathrm{T}_{\mathrm{m}}$ value of $82.5^{\circ} \mathrm{C}$ ) (Table 1 , Additional file 4). Ligand binding often stabilizes a protein and raises the $T_{m}$ value. As expected, the addition of biotin stabilized wtAvd effectively, increasing the $T_{m}$ value almost $40^{\circ} \mathrm{C}$. This $\mathrm{T}_{\mathrm{m}}$ indicates a very high binding affinity.

The effect of biotin-binding on the protein stability of steroid-binding proteins was much smaller than in the case of wtAvd, thus showing decreased biotin affinity. In fact, sbAvd-2 showed a negligible increase in $T_{m}$ in the presence of biotin $\left(\Delta \mathrm{T}_{\mathrm{m}}=0.5^{\circ} \mathrm{C}\right)$. The presence of testosterone only slightly increased the $\mathrm{T}_{\mathrm{m}}$ values of sbAvds $\left(\Delta \mathrm{T}_{\mathrm{m}}=0.6-0 \cdot 9^{\circ} \mathrm{C}\right)$. Actually, a similarly small increase was detected in the $\Delta \mathrm{T}_{\mathrm{m}}$ value of wtAvd when testosterone was added. However, because the testosterone-binding of wtAvd was not detected in other analyses (SPR and microplate analysis) the mechanism of stabilization remains unclear.

\section{Discussion}

In the present study, Avd proteins were displayed on the M13 phage in a monovalent form fused with the Cterminal region (aa residues 198-406) of the coat protein pIII. The crucial requirement for the functional display on phage is successful expression of the protein in $E$. coli. As has been earlier reported Avd protein can be efficiently expressed in a soluble form in E. coli [26]. The close relative of Avd, streptavidin, has been displayed on bacterial phages as a pVIII fusion [30,31]. However, to our best knowledge, chicken Avd has not been previously displayed on phages for screening purposes.

To evaluate the different modes for Avd display, we generated constructs expressing pIII fused to Avd and constructs that expressed free Avd subunits in addition to the pIII fusions. Based on the expression analysis, binding properties and selection experiments, the construct that expressed both fusion and free Avd showed an enhanced assembly of functional, tetrameric Avd on the phage compared to the Avd-pIII fusion alone (Additional file 2). However, the construct that expressed pIII-fused Avd was chosen for use in the selection system for the generation of gene libraries because the use of only one Avd gene in the protein display would ensure that the selected proteins could be assembled to homotetramers.

The potential of the developed platform for screening novel Avd-based receptors was investigated. Five residues in the loop between $\beta$-strands 1 and 2 of Avd were randomized to generate a population of diverse Avd genes. The gene population was screened for novel binding properties using the phage display method [23]. We observed the enrichment of Avd phages binding to testosterone-coupled BSA. The Avd variant sbAvd-1, which carried the sequence N12, R13, M14, N15, H16, was further analyzed and found to bind free testosterone with an affinity similar to BSA-conjugated testosterone. Moreover, we detected that the biotin-binding affinity of sbAvd-1 was not completely diminished; it was still high enough to inhibit sbAvd-1 from binding to testosteroneBSA (Table 1, Figures 4 and 5). This finding confirmed that testosterone occupies the same binding site in Avd as does biotin. Based on the cross-creativity measurements, sbAvd-1 also binds other steroid hormones, such as progesterone and DHEAS (Figures 4 and 5).

SbAvd-1 was further engineered to decrease biotin binding and to improve steroid specificity. From the 
phage display selections, the sbAvd-1-derived protein, named sbAvd-2, with a mutated loop between $\beta$-strands 3 and 4 was captured. This mutant showed similar or slightly decreased binding affinity towards immobilized testosterone, whereas the biotin-binding affinity was clearly decreased (Table 1, Figures 4 and 5). We also noticed that sbAvd-2 bound more tightly to free testosterone than did sbAvd-1 (Figure 5). Significantly, sbAvd2 preferred steroids as a ligand over biotin; this finding is important when considering the applications for biofluids, in which biotin is often present in relatively high concentrations.

This study and our preliminary results from experiments with a number of different target ligands (Hiltunen S, Riihimäki TA et al., unpublished data) suggest that Avd-based receptors for various different small molecules can be tailored. These Avd-based receptors may be valuable tools for diagnostic use in the future.

\section{Conclusions}

The current study provides a promising platform for the selection of tailored ligand-binders evolved from the Avd scaffold in the monovalent pIII protein display. The Avd scaffold has characteristics that are beneficial in protein engineering, such as high thermal and chemical stability, simple folding and an optimal structure for small ligands. Furthermore, Avd can be modified rather freely without major change in the fold [18]. Novel Avds that can simultaneously bind multiple ligands could become next-generation molecular tools for clinical and diagnostic applications [22,32]. Importantly, novel Avdbased receptors could be used in applications that require harsh conditions.

\section{Methods}

\section{Construction of phagemid vectors}

All of the basic recombinant DNA methods were performed essentially as previously described [33]. Appropriate restriction sites were added to the cDNA of the Avd core sequence [34] by PCR using the primers Avd_NheI_5' and Avd_NotI_3' (Additional file 5). PCR products were first subcloned into the $\mathrm{pCR}^{\circledR} 2.1$-TOPO plasmid by TOPO TA-cloning (Invitrogen) and the plasmids were transformed into $E$. coli TOP10 cells. Plasmids were isolated from colonies that contained the inserts based on the blue-white screening. Avd fragments were cut out from the $\mathrm{pCR}^{\circledR} 2.1$-TOPO-plasmid using the NheI and NotI restriction enzymes and ligated into the phagemid vector (pBluescript SK+ derived phagemid, Research Center of Finland, Biotechnology, Espoo, Finland). The cDNA of the Avd was cloned into the phagemid vector as an N-terminal fusion to the C-terminal domain (amino acids 198-406) of the minor phage coat protein III. In the Avd/Avd-pIII constructs, the coding sequence of the free Avd was subcloned using the primers Avd_NheI_5' and Avd_AscI_stop_3' (Additional file 5). The Avd-pIII expression cassette was generated by subcloning a fragment amplified from the primers Avd_SfiI_5' and Avd_NotI_3' (Additional file 5). The nucleotide sequences of the Avd constructs were verified by sequencing on an ABI PRISM 3100 Genetic Analyzer (Applied Biosystems) according to the protocols recommended by the manufacturer (ABI PRISM BigDye Terminator Cycle Sequencing Kit v.1.1, Applied Biosystems).

\section{Amplification of phage particles}

All of the basic phage display methods were performed essentially as previously described [35]. Phagemid vectors with the Avd insert were transformed into chemically competent E. coli XL1-Blue cells (Stratagene, La Jolla, CA) with the heat shock method. Phage stocks of the different Avd display constructs were made from individual colonies picked from the transformation plates into super broth (SB) medium supplemented with the appropriate antibiotics and glucose. The bacterial cultures were infected with the helper phage $\left(10^{12} \mathrm{pfu} /\right.$ ml) VCS-M13 (Stratagene, LaJolla, CA) for amplification of Avd phages. Phages were PEG precipitated and analyzed by SDS-PAGE and western blotting following immunostaining with the polyclonal rabbit $\alpha$-avd IgG (University of Oulu, 1:5000) and the monoclonal mouse anti-pIII IgG (Biosite, Sweden, 1:2000) antibodies.

\section{Functionality test of Avd-displaying phages}

The functionality of the Avd phages was tested by panning the phages on surfaces coated with BSA conjugated to HABA (HABA-BSA) [36]. As a positive control for selection, the Avd mutant N118M was also displayed on the phages. Panning was performed essentially as previously described [35]. For elution, vigorous shaking in $100 \mathrm{mM}$ hydrochloric acid containing $10 \mu \mathrm{M}$ D-biotin (Biochemica, Fluka, 14400) was performed. In total, three selection rounds were performed. After each round the integrity of the Avd expression units was analyzed by restriction enzyme digestions of the phagemid DNA samples. Importantly, both single and double Avd and $\operatorname{Avd}(\mathrm{N} 118 \mathrm{M})$ construct phages were competed against each other. Equivalent amounts of phages displaying Avd or Avd(N118M) mutant were mixed and biopanned. The ratio between constructs was determined with DNA sequencing.

\section{Construction of Avd L1,2 library}

To construct an Avd DNA library, amino acids N12, D13, L14, G15, and S16 in the loop between beta strands 1 and $2(\mathrm{~L} 1,2)$ were randomized. The libraries were constructed essentially as previously described 
[35]. A nucleic acid fragment of 105 base pairs was amplified with the primers Avd_NheI_5' and Loop 1-2 _R1_3' (Additional file 5) using wt Avd cDNA as template. Parallel to this fragment, a nucleic acid fragment with 357 base pairs was PCR-amplified with the primers Loop 1-2_R2_5' and Avd_NotI_3' (Additional file 5) using wt Avd as a template. The PCR strategy is presented in Additional file 6. The desired amplification products were separated by agarose gel electrophoresis and isolated from the gel using the Nucleo Spin Extract II kit (Macherey-Nagel) according to the manufacturer's instructions. PCR products were combined in the second amplification step in the presence of the PCR primers Avd_NheI_5' and Avd_NotI_3' (Additional file 5). This amplification resulted in a DNA fragment of 462 base pairs. The fragment was isolated from the gel and cut with the restriction enzymes NheI and NotI (Fermentas) followed by purification using the Nucleo Spin Extract II kit (Macherey-Nagel). Fragments were ligated into the phagemid vector and the resulting ligation product was transformed into electrocompetent cells of the E. coli strain XL1-Blue (Stratagene) by electroporation. Transfected bacteria were infected with the VCS-M13 helper phage (Stratagene) and phages were harvested from the culture and purified with PEG precipitation. The amount of phage particles was determined by titration.

\section{Selecting steroid-binders from the Avd L1,2 library}

The Avd L1,2 library was panned against the steroid hormone testosterone. As a negative control, wtAvd-displaying phages were also panned against testosterone. NUNC immunosorp plates were coated with a testosterone-BSA conjugate (Sigma, T-3392, 1 нg). Phages were preincubated in the BSA-coated wells to prevent nonspecific binding. Three to four selection cycles were conducted, and the stringency of washing conditions was increased every panning round to decrease nonspecific binding. In the first panning round wells were washed three times with phosphate buffered saline containing $0.05 \%$ Tween (PBS-Tween) and five times with phosphate buffered saline (PBS). Phages were eluted by vigorous shaking for 10 minutes in $100 \mathrm{mM}$ hydrochloric acid containing $10 \mu \mathrm{M}$ D-biotin (Biochemica, Fluka, 14400). Biotin was used for elution because it was probable that after randomization of the loop L1,2 the resulting Avd would still have a rather high affinity towards biotin. In the final panning round $10 \mu \mathrm{M}$ testosterone (Steraloids Inc., USA) was used in addition to $100 \mathrm{mM}$ hydrochloric acid for elution. The eluted phage solutions were neutralized with $2 \mathrm{M}$ Tris. Eluted phages were used to infect $E$. coli XL1-Blue cells and aliquots of the infected bacteria were plated to quantify the amount of eluted phages. Phages were amplified and purified as described earlier. Precipitated phages were then used for the next round of selection. After every panning round, results were verified by sequencing (20 sequences), and the number of phage particles was determined by phage titration.

\section{Generation and screening of the SbAvd-1 L3,4 library}

An SbAvd-1-derived DNA library was constructed and ligated into the phagemid as described above. In the library amino acids T35, A36, V37, and T38 in the loop between beta strands 3 and $4(\mathrm{~L} 3,4)$ were randomized; thus, in the construction of the library the primers Avd_NheI_5' and 3_4R_1_3' and primers 3_4R_2_5' and Avd_NotI_3' (Additional file 5) were used in the PCR. SbAvd-1 cDNA was used as a template. Ligated phagemid was transformed into electrocompetent cells of $E$. coli strain XL1-Blue (Stratagene) by electroporation. Transfected bacteria were infected with the VCS-M13 helper phage (Stratagene) and phages were harvested from the culture and purified with PEG precipitation. The amount of phage particles was determined by titration.

The sbAvd-1 L3,4 library was biopanned against the steroid hormone testosterone similarly as described above, with some exceptions. NUNC immunosorp plates were coated with a testosterone-BSA conjugate (Sigma, $\mathrm{T}-3392,200 \mathrm{ng})$. Phages were preincubated in BSAcoated wells before the panning procedure. Four selection cycles were performed. Phages were eluted by vigorous shaking for 10 minutes in $100 \mathrm{mM}$ hydrochloric acid containing $35 \mu \mathrm{M}$ testosterone (Steraloid Inc., USA). After each panning round, results were verified by sequencing (20 sequences), and the number of phage particles was determined by phage titration. Additionally, every panning round was screened for binders by anti-M13 as previously described [37]. For the ELISA, (NUNC) immunosorp plates were coated with a testosterone-BSA conjugate (Sigma, T-3392, $1 \mu \mathrm{g}$ ), wells were blocked with $5 \%$ milk solution, and detection was performed with Anti-M13/HRP (GE Healthcare) and read with a microplate reader (Bio-Rad $680 \mathrm{XR}$ ).

\section{Production and purification of Avd mutants}

For biochemical analyses, the proteins were produced in E. coli strain BL21-AI (Invitrogen) using expression vector pET101/D (Invitrogen) [26]. After sonication (Sonics \& Materials Vibra Cell ${ }^{\mathrm{TM}}$ ) and DNaseI (New England Bio Labs) treatment of $E$. coli cells, the purification of the proteins was conducted using Ni-NTA affinity chromatography according to the instructions of the manufacturer (QIAGEN).

The oligomeric state of the proteins was assayed with fast protein liquid chromatography (FPLC) gel-filtration using an ÄKTApurifierTM HPLC equipped with a 
Superdex 200 10/300 GL column (Tricorn, Amersham Biosciences, GE Healthcare). The column was calibrated using the gel-filtration mixture (thyroglobulin, $\gamma$-globulin, ovalbumin, myoglobin, and vitamin B12; Bio-Rad Laboratories) as a molecular-mass standard. Sodium phosphate buffer (20 mM, pH 7.4) with $1 \mathrm{M} \mathrm{NaCl}$ and, $20 \mathrm{mM}$ imidazole was used as the liquid phase. Protein samples of $90-193 \mu \mathrm{g}$ in a volume of $500 \mu \mathrm{l}$ were used in the analysis.

\section{DSC measurements of wtAvd and steroid-binding Avds}

Proteins $(0.225 \mathrm{mg} / \mathrm{ml})$ were analyzed in sodium phosphate buffer (20 mM, pH 7.4), containing $20 \mathrm{mM}$ imidazole and $1 \mathrm{M} \mathrm{NaCl}$. D-biotin (Biochemica, Fluka, 14400) and testosterone (Steraloids Inc., USA) were diluted with the measurement buffer to a final concentration of $50 \mu \mathrm{M}$. All solutions were degassed prior to measurements to avoid air bubbles. An automated capillary VP-DSC instrument (GE Healtcare, MicroCal, Northampton, USA) was used to measure the stability of the proteins with or without ligands. During the measurement, protein samples were heated from $20^{\circ} \mathrm{C}$ to $130^{\circ} \mathrm{C}$ at a scanning rate of $120^{\circ} \mathrm{C} / \mathrm{h}$. Feedback mode was set to low and the filler period was $8 \mathrm{~s}$. Temperature transition midpoints $\left(\mathrm{T}_{\mathrm{m}}\right)$ were recorded from the highest peaks and the calorimetric heat changes $(\Delta \mathrm{H})$ were calculated using the MicroCal Origin 7 software (GE Healtcare, MicroCal, Northampton, USA).

\section{Determination of ligand-binding specificity of Avd forms by microplate assay}

MaxiSorp F96 microplate wells (NUNC) were coated with $500 \mathrm{ng}$ of conjugated ligand (HSA-conjugated progesterone, hydrocortisone, testosterone, and cholic acid (Technical Research Center of Finland, Espoo, Finland); or BSA-conjugated testosterone (A6958-000, Steraloids Inc., USA), and biotin (Jenni Leppiniemi, University of Tampere, Finland)), and with $500 \mathrm{ng}$ of the carrier proteins HSA and BSA (A7906, Sigma) in $100 \mu \mathrm{l}$ of PBS for 2 hours $37^{\circ} \mathrm{C}$. Carrier proteins were used as negative controls. Plates were washed three times with PBSTween, blocked with $0.5 \%$ BSA-PBS for $30 \mathrm{~min}$, and then washed again. Proteins $(0.9 \mu \mathrm{g} / \mathrm{ml})$ in sodium phosphate buffer $(20 \mathrm{mM}, \mathrm{pH} 7.4)$ with $1 \mathrm{M} \mathrm{NaCl}$ and 20 $\mathrm{mM}$ imidazole, were added to the wells and incubated for $1 \mathrm{~h}$. A portion of wtAvd (Belovo, Bastogne, Belgium), sbAvd-1 and sbAvd-2 was preincubated with $10 \mu \mathrm{M}$ Dbiotin (Biochemica, Fluka, 14400). Bound Avd was detected by rabbit $\alpha$-avd IgG (University of Oulu) and with alkaline phosphatase conjugated goat anti-rabbit IgG (A3937, Sigma). After adding the phosphatase substrate solution ( $1 \mathrm{mg} / \mathrm{ml} \mathrm{pNPP}$ (S0942, Sigma) in 1 $\mathrm{M}$ diethanolamine $\mathrm{pH} 9.8$, with $0.5 \mathrm{mM} \mathrm{MgCl}_{2}$ ), the plates were read after 15 minutes at A405 with a microplate reader (Bio-Rad 680 XR).

\section{Biosensor analyses of steroid-binding Avds}

A BIAcore X optical biosensor (Biacore, Uppsala, Sweden) was used for the analysis of binding kinetics. Testosterone-BSA was coupled to the carboxymethylated dextran layer of a sensor chip using standard amine coupling chemistry (1000 RU, $40 \mu \mathrm{l} / \mathrm{min}$ flow rate). Samples of sbAvd-1, and sbAvd-2 were diluted in 50 $\mathrm{mM}$ sodium phosphate containing $1 \mathrm{M} \mathrm{NaCl}$, and the same buffer was used in the measurements. The binding of the sbAvd-1 and sbAvd-2 samples on testosteroneBSA coated chips was measured and the kinetic constants were determined from the measurements performed with different protein concentrations using the BiaEvaluation software according to the manufacturer's instructions. WtAvd was used as a negative control.

The binding of steroid-binding proteins to the testosterone-BSA surface was competed with free steroid hormone molecules (testosterone, DHEAS, androstenedione, estradiol, and DHT (Steraloids Inc. USA)) and free biotin (Biochemica, Fluka, 14400) to evaluate the specificity of binding. Additionally, steroidbinding was more closely detected by measuring the binding in the presence of varying concentrations $(0.75$ $\mu \mathrm{M}-50 \mu \mathrm{M})$ of inhibiting testosterone.

For the determination of biotin-binding kinetics, a sensor chip was prepared as follows: diaminoethylene was first attached to the surface using a mixture containing $0.2 \mathrm{M}$ 1-ethyl-3-(3-dimethylaminopropyl) carbodiimide hydrochloride (EDC) and $0.05 \mathrm{M} \mathrm{N}$ hydroxysuccinimide (NHS) in water. Second, to introduce amino groups to the surface, $1 \mathrm{M}$ ethylenediamine (Fluka 03550) in water was applied. Finally, $5 \mathrm{mM}$ biotin $\mathrm{N}$-succinimidyl ester (Biochemica, Fluka, 14405) in 50\% DMSO was injected on the surface $(40 \mu \mathrm{l} / \mathrm{min}$ flow rate, $\sim 130 \mathrm{RU}$; please note that the determination of the bound mass is not very accurate in case of small molecules because the immobilization can change the physicochemical properties of the surface).

\section{Molecular Recognition Force Spectroscopy experiments} Molecular recognition force spectroscopy (MRFS) was used to study the interaction of the produced proteins with testosterone and biotin. Steroid-binding proteins were covalently bound to modified mica sheets via lysines as previously described [38]. Testosterone was coupled to the AFM tip via the heterobifunctional Fmoc-PEG-NHS crosslinker as previously described 
[28], resulting in a covalent attachment of testosterone via a flexible spacer (Figure 6A).

All MRFS experiments were performed on a Pico SPM I (Agilent Technologies, Santa Clara, CA). All modified cantilevers used had nominal spring constants between 10-30 pN/nm (Veeco Instruments, Santa Barbara, CA). The effective spring constants of the cantilevers were determined by the thermal noise method $[39,40]$. Forcedistance cycles were completed using a z-range of 200 and $300 \mathrm{~nm}$. Sweep durations were adjusted between 0.25 and $4 \mathrm{~s}$. During one data set of 1000 force-distance curves, the lateral tip position was changed (a few hundred $\mathrm{nm}$ ) about every 100 curves to ensure that the binding events were statistically reasonable. The specificity of the binding was proved by adding free testosterone $(200 \mathrm{nM})$ into the measuring solution and incubating for approximately $1 \mathrm{~h}$ to block the ligandbinding sites of the proteins.

\section{Additional material}

Additional file 1: Structural comparison of Avd-BTN and Avd-HABA complexes. 3D-structures of Avd complexed with biotin (A) and HABA (B). These two ligands have different hydrogen bonding interactions with the amino acids at the binding site. (A) Hydrogen bonds formed between the ureido ring of biotin and Avd are shown here as dashed green lines. (X-ray crystallographic structure (PDB 2AVI)) [41]. (B) A significant difference between biotin and HABA binding to Avd is seen in the interaction of the ligand with asparagine 118. Although other Avd key residues interacting with the BTN ureido ring also interact with HABA, there is no hydrogen bonding partner for N118 in HABA. Again, hydrogen bonds formed between the carboxyl group of HABA and Avd are shown as dashed green lines. The loop between $\beta$-strands 3 and 4 was not resolved in the avd-HABA -complex (coordinates kindly provided by Prof. Oded Livnah). The figure was made with the VMD program [42].

Additional file 2: Sequencing results and input from the control selections. The percentage of wt Avd and Avd(N118M) mutant sequences after sequencing analysis from the different rounds of HABA selection. The amount of input phages is shown as colony forming units (cfu) per milliliter of culture.

Additional file 3: Gel-filtration chromatograms of sbAvd-1 and sbAvd-2 proteins. The chromatograms of steroid-binding proteins determined at wavelength of $280 \mathrm{~nm}$ by gel-filtration. Besides the main peak, there is also a small peak of oligomeric form of the protein in the chromatogram of sbAvd-1 protein (gray curve). It is a typically observation also in the case of wtAvd [26]. The chromatogram of sbAvd2 protein is shown with black curve. The tetrameric form of the protein is dominant in both samples.

Additional file 4: The effect of ligand-binding to the stability of wtAvd, sbAvd-1 and sbAvd-2 proteins. DSC thermograms were obtained from the protein sample $(0.225 \mathrm{mg} / \mathrm{ml})$ by scanning temperature range of $20^{\circ} \mathrm{C}$ to $130^{\circ} \mathrm{C}$ with heating rate of $120^{\circ} \mathrm{C} / \mathrm{min}$. The analysis was conducted in the absence and presence of ligands (50 $\mu \mathrm{M})$.

Additional file 5: The cloning primers used in the study. The restriction enzyme cleavage sites are indicated in italics.

Additional file 6: A schematic presentation of the construction strategy of the Avd L1,2 library. for 1-2 loop library a nucleic acid fragment of 105 base pairs was amplified (Step 1, A) with the primers Avd_Nhel_5' and Loop 1-2_R1_3' (schematically referred in the figure as Avd_mutant_3') using wtAvd cDNA as a template. Parallel to this, a nucleic acid fragment with 357 base pairs, was PCR-amplified (Step 1, B) with the primers Loop 1-2_R2_5' (schematically referred in the figure as
Avd_combine_5') and Avd_Notl_3', also using wtAvd as a template. Amplified fragments were combined in a second amplification step in the presence of PCR primers Avd_Nhel_5' and Avd_Notl_3', wherein a DNA fragment of 462 base pairs was obtained.

\section{Abbreviations}

AFM: atomic force microscopy; BSA: bovine serum albumin: BTN: D-biotin; DHEAS: dehydroepiandrosterone sulphate; DHT: dihydrotestosterone; DMSO: dimethyl sulfoxide; EDC: 1-ethyl-3-(3-dimethylaminopropyl) carbodiimide hydrochloride; HABA: 4'-hydroxyazobenzene-2-carboxylic acid; HSA: human serum albumin; MRFS: molecular recognition force spectroscopy; NHS: Nhydroxysuccinimide; PBS: phosphate buffered saline; PEG: polyethylene glycol; sbAvd: steroid-binding avidin; SB: super broth; SDS-PAGE: sodiumdodecyl sulfate polyacrylamide gel electrophoresis; wtAvd: wild type avidin.

\section{Acknowledgements}

We thank the laboratory staff of VTT (Technical Research Center of Finland) Biotechnology, Espoo, Finland, for excellent technical assistance. We thank MSc. Antti Tullila for kindly providing us HSA-conjugates. We also thank the staff at the Molecular Biology group, Department of Biological and Environmental Science, University of Jyväskylä for their assistance. We thank MSc. Sampo Kukkurainen for help in molecular modeling. We would also like to thank MSc. Kaisa Helttunen, Professor Kari Rissanen and Dr. Juhani Huuskonen for their help in the preparation of the HABA-BSA conjugate. We thank Hong Chang for participation in the experimental work. The study was financially supported by the Academy of Finland (115976, 121236); the National Technology Agency of Finland (BioFace 40055/08); European Micro and Nano Technology support program (FFG 421695); Pirkanmaa Hospital District and Tampere Graduate Program in Biomedicine and Biotechnology.

\section{Author details}

${ }^{1}$ Institute of Biomedical Technology, University of Tampere and Tampere University Hospital, Fl-33520 Tampere, Finland. ${ }^{2}$ Institute of Biophysics, Johannes Kepler University Linz, 4040 Linz, Austria. ${ }^{3}$ VTT Technical Research Centre of Finland, FI-02044 VTT, Finland.

\section{Authors' contributions}

TAR conceived the study, constructed the Avd libraries, performed most of the experiments, and contributed to the writing of the manuscript; $\mathrm{SH}$ participated in the construction of the Avd libraries and in the experimental work and contributed to the writing of the manuscript; JAEM participated in the experimental work and contributed to the writing of the manuscript; MR, AE and PH performed MRFM measurements and contributed to the writing of the manuscript; HRN, MSK and KT conceived the study and supervised the work; VPH conceived and designed the study, supervised the work and contributed to the writing of the manuscript. All authors read and approved the final manuscript.

Received: 29 April 2011 Accepted: 9 June 2011 Published: 9 June 2011

\section{References}

1. Hoogenboom HR: Selecting and screening recombinant antibody libraries. Nat Biotechnol 2005, 23:1105-1116.

2. Hoess RH: Protein design and phage display. Chem Rev 2001, 101(10):3205-3218

3. Sarikaya M, Tamerler C, Jen AK, Schulten K, Baneyx F: Molecular biomimetics: nanotechnology through biology. Nat Mater 2003, 2:577-585

4. Binz HK, Amstutz P, Pluckthun A: Engineering novel binding proteins from nonimmunoglobulin domains. Nat Biotechnol 2005, 23:1257-1268.

5. Skerra A: Alternative non-antibody scaffolds for molecular recognition. Curr Opin Biotechnol 2007, 18:295-304.

6. Werner RG: Economic aspects of commercial manufacture of biopharmaceuticals. J Biotechnol 2004, 113:171-182.

7. Hudson PJ, Souriau C: Engineered antibodies. Nat Med 2003, 9:129-134.

8. Saerens D, Ghassabeh GH, Muyldermans S: Single-domain antibodies as building blocks for novel therapeutics. Curr Opin Pharmacol 2008, 8:600-608 
9. Nygren PA, Skerra A: Binding proteins from alternative scaffolds. $J$ Immunol Methods 2004, 290:3-28.

10. Gebauer M, Skerra A: Engineered protein scaffolds as next-generation antibody therapeutics. Curr Opin Chem Biol 2009, 13:245-255.

11. Beste G, Schmidt FS, Stibora T, Skerra A: Small antibody-like proteins with prescribed ligand specificities derived from the lipocalin fold. Proc Natl Acad Sci USA 1999, 96:1898-1903.

12. Schlehuber S, Skerra A: Tuning ligand affinity, specificity, and folding stability of an engineered lipocalin variant - a so-called 'anticalin' using a molecular random approach. Biophys Chem 2002, 96:213-228.

13. Laitinen $\mathrm{OH}$, Nordlund HR, Hytönen VP, Kulomaa MS: Brave new (strept) avidins in biotechnology. Trends Biotechnol 2007, 25:269-277.

14. Green NM: Avidin. Adv Protein Chem 1975, 295:85-133.

15. Repo S, Paldanius TA, Hytönen VP, Nyholm TK, Halling KK, Huuskonen J, Pentikäinen OT, Rissanen K, Slotte JP, Airenne TT, Salminen TA, Kulomaa MS, Johnson MS: Binding Properties of HABA-Type Azo Derivatives to Avidin and Avidin-Related Protein 4. Chem Biol 2006, 13:1029-1039.

16. Livnah O, Bayer EA, Wilchek M, Sussman JL: Three-dimensional structures of avidin and the avidin-biotin complex. Proc Natl Acad Sci USA 1993, 90297:5076-5080.

17. Rosano C, Arosio P, Bolognesi M: The X-ray three-dimensional structure of avidin. Biomol Eng 1999, 16:5-12.

18. Laitinen OH, Hytönen VP, Nordlund HR, Kulomaa MS: Genetically engineered avidins and streptavidins. Cell Mol Life Sci 2006, 63:2992-3017.

19. Nordlund HR, Laitinen OH, Hytönen VP, Uotila ST, Porkka E, Kulomaa MS Construction of a dual chain pseudotetrameric chicken avidin by combining two circularly permuted avidins. J Biol Chem 2004, 279:36715-36719.

20. Hytönen VP, Nordlund HR, Hörhä J, Nyholm TK, Hyre DE, Kulomaa T, Porkka EJ, Marttila AT, Stayton PS, Laitinen OH, Kulomaa MS: Dual-affinity avidin molecules. Proteins 2005, 61:597-607.

21. Riihimäki TA, Kukkurainen S, Varjonen S, Hörhä J, Nyholm TKM, Kulomaa MS Hytönen VP: Construction of chimeric dual-chain avidin by tandem fusion of the related avidins. PLOS One 2011, 6:e20535.

22. Nordlund HR, Hytönen VP, Hörhä J, Maatta JA, White DJ, Halling K, Porkka E, Slotte JP, Laitinen $\mathrm{OH}$, Kulomaa MS: Tetravalent single chain avidin: From subunits to protein domains via circularly permuted avidins. Biochem J 2005, 392:485-491.

23. Smith GP: Filamentous fusion phage: novel expression vectors that display cloned antigens on the virion surface. Science 1985, 228:1315-1317.

24. Hoogenboom HR, Griffiths AD, Johnson KS, Chiswell DJ, Hudson P, Winter G: Multi-subunit proteins on the surface of filamentous phage: methodologies for displaying antibody (Fab) heavy and light chains. Nucleic Acids Res 1991, 19:4133-4137.

25. Määttä JA, Airenne TT, Nordlund HR, Jänis J, Paldanius TA, Vainiotalo P, Johnson MS, Kulomaa MS, Hytönen VP: Rational modification of ligandbinding preference of avidin by circular permutation and mutagenesis. Chembiochem 2008, 9:1124-1135.

26. Hytönen VP, Laitinen $\mathrm{OH}$, Airenne $\Pi$, Kidron $H$, Meltola NJ, Porkka E, Hörhä J, Paldanius T, Määttä JA, Nordlund HR, Johnson MS, Salminen TA, Airenne KJ, Ylä-Herttuala S, Kulomaa MS: Efficient production of active chicken avidin using a bacterial signal peptide in Escherichia coli. Biochem J 2004, 384:385-390.

27. Hinterdorfer P, Dufrene YF: Detection and localization of single molecular recognition events using atomic force microscopy. Nat Methods 2006, 3:347-355.

28. Wildling $L$, Hinterdorfer $P$, Kusche-Vihrog $K$, Treffner $Y$, Oberleithner $H$ : Aldosterone receptor sites on plasma membrane of human vascular endothelium detected by a mechanical nanosensor. Pflugers Arch 2009, 458:223-230.

29. Baumgartner $W$, Hinterdorfer $P$, Schindler H: Data analysis of interaction forces measured with the atomic force microscope. Ultramicroscopy 2000, 82:85-95.

30. Avrantinis SK, Stafford RL, Tian X, Weiss GA: Dissecting the streptavidinbiotin interaction by phage-displayed shotgun scanning. Chembiochem 2002, 3:1229-1234.

31. Sidhu SS, Weiss GA, Wells JA: High copy display of large proteins on phage for functional selections. J Mol Biol 2000, 296:487-495.
32. Leppiniemi J, Määttä JA, Hammaren H, Soikkeli M, Laitaoja M, Jänis J, Kulomaa MS, Hytönen VP: Bifunctional avidin with covalently modifiable ligand binding site. PLoS One 2011, 6:e16576.

33. Sambrook J, Fritsch EF, Maniatis T: Molecular Cloning: A Laboratory Manual. Cold Spring Harbor Laboratory Press, Cold Spring Harbor, NY; 1990.

34. Gope ML, Keinänen RA, Kristo PA, Conneely OM, Beattie WG, ZaruckiSchulz T, O'Malley BW, Kulomaa MS: Molecular cloning of the chicken avidin cDNA. Nucleic Acids Res 1987, 15:3595-3606.

35. Barbas CF III, Burton DR, Scott JK, Silverman GJ: Phage Display, A Laboratory manual. Cold Spring Harbor Laboratory Press, Cold Spring Harbor, NY; 2001.

36. Hofstetter H, Morpurgo M, Hofstetter O, Bayer EA, Wilchek M: A labeling, detection, and purification system based on 4-hydroxyazobenzene-2carboxylic acid: an extension of the avidin-biotin system. Anal Biochem 2000, 284:354-366

37. Kingsbury GA, Junghans RP: Screening of phage display immunoglobulin libraries by anti-M13 ELISA and whole phage PCR. Nucleic Acids Res 1995, 23:2563-2564.

38. Kamruzzahan AS, Kienberger F, Stroh CM, Berg J, Huss R, Ebner A, Zhu R, Rankl C, Gruber HJ, Hinterdorfer P: Imaging morphological details and pathological differences of red blood cells using tapping-mode AFM. Biol Chem 2004, 385:955-960.

39. Hutter JL, Bechhoefer J: Calibration of atomic-force microscope tips. Review of Scientific Instruments 1993, 64:1868-1873.

40. Butt HJ, Jaschke M: Calculation of thermal noise in atomic force microscopy. Nanotechnology 1995, 6:1-7.

41. Livnah O, Bayer EA, Wilchek M, Sussman JL: Three-dimensional structures of avidin and the avidin-biotin complex. Proc Natl Acad Sci USA 1993, 90:5076-5080.

42. Humphrey W, Dalke A, Schulten K: VMD: visual molecular dynamics. J Mol Graph 1996, 14:33-8, 27-8.

doi:10.1186/1472-6750-11-64

Cite this article as: Riihimäki et al:: Modification of the loops in the ligand-binding site turns avidin into a steroid-binding protein. BMC Biotechnology 2011 11:64.

\section{Submit your next manuscript to BioMed Central and take full advantage of:}

- Convenient online submission

- Thorough peer review

- No space constraints or color figure charges

- Immediate publication on acceptance

- Inclusion in PubMed, CAS, Scopus and Google Scholar

- Research which is freely available for redistribution

Submit your manuscript at www.biomedcentral.com/submit
C) Biomed Central 